\title{
Forecasting Admissions in COMSATS Institute of Information Technology, CIIT, Islamabad
}

\author{
Erum Rehman and Dr. Noor. M. Larik
}

\begin{abstract}
The increasing competition for admissions in Higher Education Institutes for admissions has become a problem for Higher Education Institutes for making adequate policies for decision making. The COMSATS Institute of Information Technology has eight campuses all over the country and headquarters in Islamabad, Pakistan. Due to its popularity and ranking first in the country as institute of science and technology and sixth in all general universities of country. There has been in general significant increase of the students for admissions. Thus it requires future demand for admissions to make adequate policies for students as well as faculty requirement. In this article, an attempt has been made to forecast admissions of students particularly in main campus located in Islamabad by using models such as Holts linear trend model, simple linear regression model and simple linear model, it has been concluded that simple linear regression model gives us more accurate results as compared to others.
\end{abstract}

\section{Introduction}

A forecast is an estimate of uncertain future events (literally, to "cast forward" by extrapolating from past and current data). It is an important tool for policy and decision making that helps management in its attempts to cope up with the uncertainty of the future, relying mainly on data from the past and present and analysis of trends. Forecasting starts with certain assumptions based on the management's experience, knowledge, and judgement. Forecasts are used to improve decision making and planning. Even though forecasts are almost always in error, it is better to have the limited information provided by a forecast than to make decisions in total ignorance about the future.

\section{Forecasting Future Enrollments in Admission}

An enrollment model is a technique for estimating how future enrollments will be affected by alternate enrollment policies. Enrollment management most often takes the form of regulating the number and types of new students who will be enrolled in university level. Any higher education institute like a university should have a tentative future plan. The first step in establishing such a plan is to get the required information concerning the projected student enrollment for the next few years. Though an enrollment forecasting is a specific type of estimation, one that uses past information in some enrollments manner to arrive at an estimate of future.

Forecasting techniques can be categorized in two broad categories:

(1) Quantitative forecasting techniques

(2) Qualitative forecasting Techniques.

\section{a. Qualitative forecasting techniques}

- Subjective, based on the opinion and judgment of policy makers, managers, decision makers and 
researchers

- Appropriate when past data are unavailable (new product) or when past data are not reliable predictors of the future

- Usually applied to intermediate

- Long range decisions

b. Quantitative forecasting techniques

- Explicit mathematical models are used to estimate future prediction of admissions as a function of past data

- Appropriate when past data are available and also are reliable predictors of the future

- Usually applied to short

- Intermediate range decisions

\section{c. Necessary conditions for a forecasting}

- Regularities should be captured

- These regularities should be informative for the future

- And they should be included in the model

- Non-regularities should be excluded

- The rest two properties are valid for all forecast methods. The latter two depend on the method that is being used.

\section{Literature Review}

D.Beck (2009) categorized the students into many categories and worked on campus level enrollment forecasting. The campus level enrollment model has been used for each category of students. He has used an exponential model for forecasting enrollments in each category. Various offices are authorized to admit undergraduate and graduate students into the university.

Janet ward (2007) has used various models for forecasting enrollment of students and has concluded that it is complex process that requires an understanding of institutional enrollment goals as well as past and current strategies employed to realize the goals, all while developing models that reliably project future enrollment. Models should be constructed based on historical data with this information placed into context. The most effective models emerge when changes in trends can be explained by changes in the surrounding competitive higher education environment (e.g., understanding why students chose to attend a competitor) or changes within the institution (e.g., understanding how a new financial aid strategy impacted yield rates). Overall, it is important to appreciate that enrollment forecasting is an iterative and collaborative process, with models being annually reviewed and refined based on feedback from various constituents within the community.

Rob J Hyndman and George Athanasopoulo (2012) forecasting: principles and practice in this book, various methods of forecasting have been described and is very useful as reference book for forecasting purposes.

\section{Methodology}

Forecasting future admissions for COMSATS Institute of Information Technology (CIIT) which was established in 1998 needs future policies/decisions for necessary actions/directions.

In this research article we have made an attempt to forecast admissions for CIIT using available data from 
(1999-2011) using Quantitative models. The methodology/models used is based on literature available on internet as mention in the literature review section.

Simple linear regression. In statistics, simple linear regression is the least squares estimator of a linear regression model with a single explanatory variable. In other words, simple linear regression fits a straight line through the set of $n$ points in such a way that makes the sum of squared residuals of the model (that is, vertical distances between the points of the data set and the fitted line) as small as possible. Suppose there are $\mathrm{n}$ data points $y_{i}, x_{i}$, where $i=1,2, n$.

$$
\mathrm{Y}=\alpha+\beta \mathrm{x}+\varepsilon
$$

Where the parameters $\beta_{0}$ and $\beta_{1}$ determine the intercept and the slope of the line respectively. And the error term does not imply a mistake, but a deviation from the underlying straight line model. It captures anything that may affect $y_{i}$ other than $x_{i}$.

We assume that these errors:

- have mean zero; otherwise the forecasts will be systematically biased.

- are not auto correlated; otherwise the forecasts will be inefficient as there is more information to be exploited in the data.

- are unrelated to the predictor variable; otherwise there would be more information that should be included in the systematic part of the model.

Linear trend model. The forecasting equation for the linear trend model is

$$
\mathrm{Y}(\mathrm{t})=\alpha+\beta \mathrm{t}
$$

Where $\mathrm{t}$ is the time index. The parameters $\alpha$ and $\beta$ (the "intercept" and "slope" of the trend line)are usually estimated via a simple regression in which $\mathrm{Y}$ is the dependent variable and the time index $\mathrm{t}$ is the independent variable. Trend Analysis is the practice of collecting information and attempting to spot a pattern, or trend, in the information. In some fields of study, the term "trend analysis" has more formally defined meanings. Although trend analysis is often used to predict future events, it could be used to estimate uncertain events in the past.

In statistics, trend analysis often refers to techniques for extracting an underlying pattern of behavior in a time series which would otherwise be partly or nearly completely hidden by noise. Trend is also defined as 'long term' movement in a time series without calendar related and irregular effects, and is a reflection of the underlying level. It is the result of influences such as population growth, price inflation and general economic changes.

Holts linear trend model. Holt (1957) extended simple exponential smoothing to allow fore-casting of data with a trend. This method involves a forecast equation and two smoothing equations (one for the level and one for the trend):

Forecast equation $\hat{y}_{t}+h \mid t=l_{t}+h b_{t}$ Level equation $l_{t}=\alpha y_{t}+(1-\alpha)\left(l_{t-1}+b_{t-1}\right)$

Trend equation $b_{t}=\beta^{*}\left(l_{t}-l_{t-1}\right)+\left(1-\beta^{*}\right) b_{t-1}$

where $l_{t}$ denotes an estimate of the level of the series at time $t, b_{t}$ denotes an estimate of the trend (slope) of the series at time $t, \alpha$ is the smoothing parameter for the level, $0 \leq \alpha \leq 1$ and $\beta^{*}$ is the smoothing parameter for the trend, $0 \leq \beta^{*} \leq 1$, we set $l_{0}=y_{1}$ and $b_{0}=y_{2}-y_{1}$. 


\section{Forecasting Results}

The results are based on the software excel and are given in table below:

Table 1

Shows actual admissions and forecasting of simple linear regression

\begin{tabular}{lllll}
\hline years & students in Islamabad campus $y$ & $\begin{array}{c}\hat{y}=\alpha+\beta x \\
\text { islamabad campus }\end{array}$ & \multicolumn{1}{c}{$\beta$} \\
\hline $1999-2000$ & 98 & 126.2307692 & -1030.77 & -28.2308 \\
$2000-2001$ & 193 & 313.6433566 & -843.357 & -120.643 \\
$2001-2002$ & 416 & 501.055944 & -655.944 & -85.0559 \\
$2002-2003$ & 911 & 688.4685315 & -468.531 & 222.5315 \\
$2003-2004$ & 1198 & 875.8811189 & -281.119 & 322.1189 \\
$2004-2005$ & 866 & 1063.293706 & -93.7063 & -197.294 \\
$2005-2006$ & 920 & 1250.706294 & 93.70629 & -330.706 \\
$2006-2007$ & 1440 & 1438.118881 & 281.1189 & 1.881119 \\
$2007-2008$ & 1973 & 1625.531469 & 468.5315 & 347.4685 \\
$2008-2009$ & 1870 & 1812.944056 & 655.9441 & 57.05594 \\
$2009-2010$ & 1921 & 2000.356643 & 843.3566 & -79.3566 \\
$2010-2011$ & 2078 & 2187.769231 & 1030.769 & -109.769 \\
$2011-2012$ & & 2375.181818 & 1218.182 & \\
$2012-2013$ & & 2562.594406 & 1405.594 & 1499.301 \\
$2013-2014$ & & 2656.300699 & 1593.007 & 1686.713 \\
$2014-2015$ & & 2750.006993 & 2843.713287 &
\end{tabular}

Table 2

Shows actual admissions and forecasting of Linear Trend Model

\begin{tabular}{llll}
\hline years & students in Islamabad campus $y$ & $t$ & $\hat{y}$ \\
\hline $1999-2000$ & 98 & 1 & 180.071 \\
$2000-2001$ & 193 & 2 & 360.142 \\
$2001-2002$ & 416 & 3 & 540.213 \\
$2002-2003$ & 911 & 4 & 720.284 \\
$2003-2004$ & 1198 & 5 & 900.355 \\
$2004-2005$ & 866 & 6 & 1080.426 \\
$2005-2006$ & 920 & 7 & 1260.497 \\
$2006-2007$ & 1440 & 8 & 1440.568 \\
$2007-2008$ & 1973 & 9 & 1620.639 \\
$2008-2009$ & 1870 & 10 & 1800.71 \\
$2009-2010$ & 1921 & 11 & 1980.781 \\
$2010-2011$ & 2078 & 12 & 2160.852 \\
$2011-2012$ & & 13 & 2340.923 \\
$2012-2013$ & & 14 & 2520.994 \\
$2013-2014$ & & 15 & 2701.65 \\
$2014-2015$ & & 16 & 2881.136 \\
$2015-2016$ & & 17 & 3061.207 \\
\hline
\end{tabular}


Table 3

Shows actual admissions and forecasting of Holts Linear Trend Model

\begin{tabular}{llllll}
\hline years & students in Islamabad campus $y$ & $t$ & levell $_{t}$ & $b_{t}$ & $\hat{y}=y_{t}+b_{t}$ \\
\hline $1999-2000$ & 98 & 0 & 98 & 95 & 193 \\
$2000-2001$ & 193 & 1 & 117 & 79.8 & 196.8 \\
$2001-2002$ & 416 & 2 & 193.76 & 79.192 & 272.952 \\
$2002-2003$ & 911 & 3 & 387.3904 & 102.0797 & 489.4701 \\
$2003-2004$ & 1198 & 4 & 826.694 & 169.5245 & 996.2185 \\
$2004-2005$ & 866 & 5 & 1157.644 & 201.8095 & 1359.453 \\
$2005-2006$ & 920 & 6 & 964.6906 & 122.857 & 1087.548 \\
$2006-2007$ & 1440 & 7 & 953.5095 & 96.04937 & 1049.559 \\
$2007-2008$ & 1973 & 8 & 1361.912 & 158.52 & 1520.432 \\
$2008-2009$ & 1870 & 9 & 1882.486 & 230.9309 & 2113.417 \\
$2009-2010$ & 1921 & 10 & 1918.683 & 191.9841 & 2110.668 \\
$2010-2011$ & 2078 & 11 & 1958.934 & 161.6373 & 2120.571 \\
$2011-2012$ & & 12 & 2086.514 & 154.826 & 2241.34 \\
$2012-2013$ & & 1 & & & 2396.166 \\
$2013-2014$ & & 2 & 3 & & 2560.992 \\
$2014-2015$ & & 4 & & & 2705.818 \\
$2015-2016$ & & 5 & & & 2860.644 \\
\hline
\end{tabular}

\section{Conclusions}

The purpose of this work is to forecast admissions in the university. Effort has been made to use various models such linear trend model, Holt trend linear model and simple linear regression. From the results it appears that models 3 i.e. simple linear regression provides reasonably accurate forecast as compared to linear trend model and Holt trend linear model. The comparison is based on variance of the actual values to the forecasted values.

\section{References}

Hyndman, R.J, \& Athanasopoulo, G., "Forecasting: principles and practice",2012.

Ward, J.,"Forecasting Enrollment to Achieve Institutional Goals", 2007.

Brockwell, P. J. \& Davis, R. A., "Time Series: Theory and Methods", 1987.

Bowerman, B. L. \& OConnell, R. T., "Forecasting and Time Series: an Applied Approach (3rd ed.)", 1993.

Tsui, P., Murdock, T., \& Mayer, L., "Trend analysis and enrollment management", 1997. 